\title{
Types of error in free recall
}

F. I. M. CRAIK

BIRKBECK COLLEGE, UNIVERSITY OF LONDON, ENGLAND

If the free recall of unrelated word lists depends on one memory store, then although the absolute number of errors may change as recall proceeds there is no reason for the relative proportions of different types of error to change. On the other hand, if recall consists of output from a short-term store which employs acoustic coding, followed by retrieval from a long-term store with different characteristics, a change in the proportions of errors would be expected. The results support the latter proposition.

Melton (1963) has argued for a single mechanism underlying short-term and long-term memory for verbal material. His argument was based in part on the apparently similar effects of interference over short and long retention intervals. Since then it has been suggested (Waugh \& Norman, 1965; Atkinson \& Shiffrin, 1967) that independent short-term and longterm stores exist but that both stores contribute to the output after short-term retention. Since some material is retrieved from long-term store (LTS) after both long and short intervals, recall will reflect the characteristics of that store in both cases.

Evidence supporting the existence of two memory stores has come from the observation that a large number of errors after short-term retention are acoustically similar to correct stimuli (Conrad, 1964). Baddeley (1966) has since shown that the difficulty involved in short-term storage of acoustically similar material does not apply to longer-term retention. Also, Conrad (1967) reported an experiment in which Ss attempted to recall four consonants after either 2.4 or $7.2 \mathrm{sec}$ of digit naming. He found that errors tended to be acoustically similar to correct letters for both retention intervals, but the distribution of errors tended more towards random for the longer interval. These findings suggest that verbal material is coded in a short-term store (STS) on an acoustic basis but that this type of coding is not so important in $\mathrm{LTS}$.

In the present experiment, the types of error in free recall of word lists were examined. If only one storage system is involved as Melton suggested, then although the absolute number of errors might change as recall proceeds, there is no reason to suppose that the relative proportions of various classes of error should change. On the other hand, if free recall is a two-stage process (Atkinson \& Shiffrin, 1967) in which Ss first read out the remaining material from a limited capacity STS whose items are coded acoustically and then search through the relevant area of a long-term verbal store with different coding characteristics, it might be expected that the first errors would be largely acoustic but that this proportion would drop as recall proceeded. On the assumption that material is lost from STS within 30 sec or so (Atkinson \& Shiffrin, 1967), errors which are intrusions from previous lists have probably arisen from LTS. If the two-stage retrieval model is correct, this type of error should be more probable in later responses. Method

Twenty Ss heard 25 lists of words presented at a rate of 48 words per min and attempted to recall each list verbally immediately after presentation. The lists were $6,9,12,15$ or 18 words in length and were composed of 1-,2-,3-or 4-syllable high frequency words or 2-syllable low frequency words. The different list lengths and types of word were not distinguished for the present analysis which dealt simply with the total number of errors from all 500 lists.

Errors were classified into four types: (1) Previous List Intrusions-words which had occurred in a previous presentation list or as erroneous responses to a previous list. (2) Acoustic-words having at least half their phonemes identical to a word in the current presentation list. (3) Semantic-words which were synonyms of a word in the current presentation list or strong associates of a presented word. (4) Randomother errors which did not fall unequivocally into one of the previous classes.

If an error fitted the definition of more than one class, the order of precedence was $1,2,3,4$. Thus an error which was both acoustically and semantically related to a correct word was classified as acoustic. This order of precedence favors objectivity.

The output position (i.e., whether the error was the first, second, third, . . . . etc. response) was recorded, and the latency with which each error followed the recall signal was subsequently measured from a tape recording of the S's responses.

\section{Results}

Errors were grouped into 2-sec intervals measured from the recall signal. In the first five intervals there were $45,64,60,47$, and 31 errors respectively; after $10 \mathrm{sec}$ the intervals did not contain sufficient errors to calculate meaningful proportions so the remaining 156 errors were combined in an "After 10 " category. Figure 1 shows that over the first 8 or 9 sec the proportion of acoustic errors falls while the proportions of other errors rise.

The errors were also analyzed by output position. The percentages of each type of error are shown in Table 1. Again acoustic errors form the bulk of the errors for the early output positions, but the proportion of this type of error falls and other proportions rise 


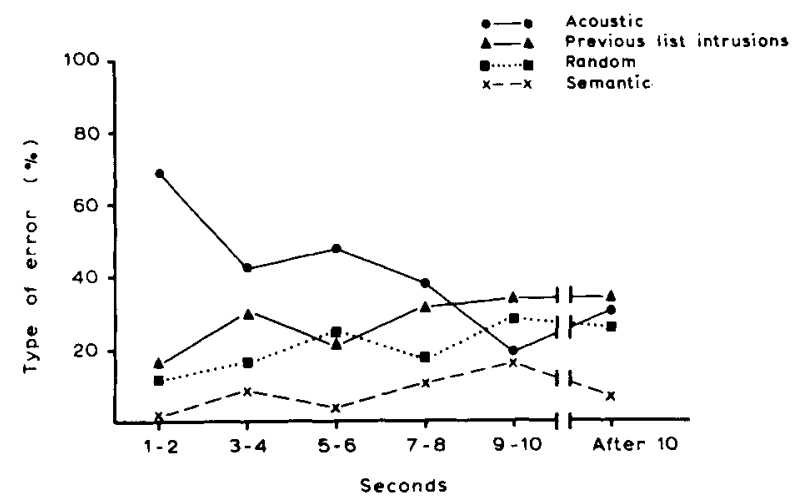

Fig. 1. Percentages of each type of error as a function of time from the start of recall.

until from position 6 the proportions are relatively stable. Acoustic errors and previous list intrusions were considered to be the least ambiguous products of STS and LTS respectively. When these errors were classified for each $S$ into responses from positions 1-5 or $6+$, it was found that 94 acoustic errors occurred in positions 1-5 while 61 occurred in position 6 or later; there were 50 previous list intrusions in positions 1-5 and 70 in 6+. A two-factor analysis of variance was carried out on these data, treating type of error as one factor and output position as the other. This analysis yielded a significant interaction between the factors $(F=8.04, \mathrm{df}=1 / 19, \mathrm{p}<.02)$. It may thus be concluded that there is a reliable tendency for the relative proportions of these types of error to change with output position.

Table 1.

Percentages of Each Type of Error at Each Output Position.

\begin{tabular}{lrrrrrrrr} 
& \multicolumn{8}{c}{ Output position } \\
\cline { 2 - 9 } Type of error & $1+2$ & 3 & 4 & 5 & 6 & 7 & 8 & $9+$ \\
\hline Acoustic & 63 & 69 & 39 & 44 & 25 & 31 & 29 & 31 \\
Previous List Intrusion & 17 & 19 & 33 & 28 & 43 & 27 & 29 & 29 \\
Semantic & 0 & 6 & 6 & 12 & 3 & 16 & 12 & 5 \\
Random & 21 & 6 & 22 & 16 & 28 & 27 & 31 & 34 \\
\hline Total no. of errors & 24 & 36 & 51 & 80 & 67 & 45 & 42 & 58 \\
\hline
\end{tabular}

\section{Discussion}

The results support the notion that two memory stores are involved in the immediate free recall of word lists. The first five responses take $8-9 \mathrm{sec}$ and appear to be drawn largely from an STS whose coding characteristics are primarily acoustic; subsequent responses are retrieved for the most part from an LTS in which semantic coding plays a greater part and where representation of words can remain active and be retrieved several lists after their presentation.

If material in STS is lost within $30 \mathrm{sec}$ or so, then the presence of previous list intrusions in the first few responses must mean either that some of these errors have been misclassified or that on some trials Ss retrieve even the first responses from LTS. In fact there may well be some misclassification of errors, but there is no reason to suppose that any bias which may exist should change over output positions or time. Similarly, it is possible that some acoustic errors are errors of perception rather than memory but again there is no reason why such misperceptions should occur primarily as initial responses. The continuing presence of acoustic errors in later output positions may reflect the base-line for such perceptual errors. From observations of the tip-of-the-tongue phenomenon (Brown \& McNeill, 1966) it seems likely, however, that LTS employs acoustic coding to some extent and that these errors are, in part at least, errors of storage or retrieval.

\section{References}

ATKINSON, R. C. \& SHIFFRIN, R. M. Human memory: a proposed system and its control processes. Technical Report 110 , Institute for Mathematical Studies in the Social Sciences, Stanford University, 1967.

BADDELEY, A. D. The influence of acoustic and semantic similarity on long-term memory for word sequences. Quart. J. exp. Psychol, 1966, 18, 302-309.

BROWN, R., \& McNEILL, D. The "tip of the tongue" phenomenon. $J$. verbal Learn. verbal Behav., 1966, 5, 325-337.

CONRAD, R. Acoustic confusions in immediate memory. Brit. J. Psychol, 1964, 55, 75-84.

CONRAD, R. Interference or decay over short retention intervals? $J$. verbal Learn. verbal Behav., 1967, 6, 49-54.

MELTON, A. W. Implications of short-term memory for a general theory of memory. J. verbal learn. verbal Behav., 1963, 2, 1-21.

WAUGH, N. C., \& NORMAN, D. A. Primary memory. Psychol. Rev.., $1965,72,89-104$ 